Service social

\title{
R. 우.S.E. du Nord : une pratique de conscientisation avec des femmes de classe populaire
}

\section{Jocelyne Barnabé}

Volume 34, numéro 2-3, 1985

L'organisation communautaire

URI : https://id.erudit.org/iderudit/706271ar

DOI : https://doi.org/10.7202/706271ar

Aller au sommaire du numéro

Éditeur(s)

École de service social de l'Université Laval

ISSN

1708-1734 (numérique)

Découvrir la revue

Citer cet article

Barnabé, J. (1985). R. ‥S.E. du Nord : une pratique de conscientisation avec des femmes de classe populaire. Service social, 34(2-3), 249-268.

https://doi.org/10.7202/706271ar d'utilisation que vous pouvez consulter en ligne.

https://apropos.erudit.org/fr/usagers/politique-dutilisation/ 


\section{R.Q.S.E. du Nord : \\ une pratique de conscientisation avec des femmes de classe populaire}

\section{Jocelyne Barnabé}

Marguerite, Jacqueline, Jeannette, Marlène, Yolande, Nicole, Gemma, Violette, Carmelle et les autres... "les femmes du Nord" comme on s'appelle. Des femmes bien différentes en apparence: entre 22 et 61 ans, deux d'entre elles seulement n'ont pas d'enfants, les autres : une en a deux, deux autres trois, puis quatre, cinq, six, neuf et... quinze enfants. Elles sont mariées, divorcées, veuves ou célibataires. Certaines habitent au Nord, en milieu semi-rural, dans la maison familiale ou en H.L.M.; d'autres en banlieue, dans un des H.L.M. construits en retrait des grands centres ou dans un petit appartement partagé, ou encore dans la « résidence familiale» déclarée comme telle au moment du divorce. Une grande ressemblance: toutes sont sans emploi, c'est-à-dire qu'elles travaillent toutes mais n'ont pas de revenu d'emploi; elles vivent de l'aide sociale, de prestations de chômage, d'une petite pension alimentaire comblée par le Bien-être social ou des rentes du mari décédé.

D'autres ressemblances aussi, plus subtiles, surtout d'ordre culturel... des ressemblances de femmes de classe populaire, base des intérêts qu'elles ont à se regrouper, à partager et à vivre des changements individuels et collectifs.

C'est avec ces femmes que je partage l'expérience de "R.Q.S.E. du Nord" depuis deux ans, comme travailleuse communautaire d'abord, puis comme organisatrice communautaire au C.L.S.C. La Source, dans la banlieue nord de Québec. R.Q.S.E. du Nord, c'est le Regroupement des femmes sans emploi du Nord de Québec. 


\section{L'intervenante en situation}

À l'entrevue de sélection où je me suis présentée pour le poste de travailleuse communautaire au C.L.S.C. La Source, en décembre 1982, j'ai eu à définir l'approche de conscientisation que je privilégiais, à circonscrire les rôles, pouvoirs et limites des intervenantes et intervenants dans les institutions et à afficher mes options pour la lutte des classes et celle des femmes.

\section{La conscienfísation et la vie}

Je considérais et je considère encore que les expériences de la vie privée furent, pour moi, autant d'éléments importants dans ma formation que les expériences "mesurables» de travail et d'études: les années de "maternitude" et de rupture avec le marché du travail, doublées d'expériences d'engagement social dans les garderies et comités d'école, un retour aux études comportant la rédaction d'une thèse menée de front avec une troisième maternité, la réintégration du marché du travail à coups de "contrats divers", en même temps qu'une séparation mouvementée entraînant des changements secondaires radicaux. Diverses expériences humaines insécurisantes, vécues hors des plans de carrière super-valorisants et tracés dans le "droit sillon", si elles sont analysées au cœur de l'action, sont indispensables à la capacité de mettre en action et de poursuivre, dans le doute et la passion, des expériences de la nature de celle qui se vit à R.Q.S.E. du Nord. C'est d'ailleurs à travers ces expériences de vie que je me suis préparée, sans le savoir, à rencontrer la conscientisation. Pour moi, ce ne fut pas tout à fait une découverte: plutôt un nouveau nom et l'occasion de théoriser et surtout d'approfondir et de confronter une façon d'aborder la vie et le travail social, façon que j'avais déjà explorée intuitivement dans l'enseignement à la maternelle et dans le reste de mon rapport au monde. Ce n'était pas un choix mais une nécessité, un «allant de soi ". II allait en effet de soi pour moi, autant que je me souvienne, que je travaillerais à réduire les inégalités sociales. Il allait de soi aussi que je ne serais jamais une savante, c'est-à-dire celle qui sait et dit la vérité, et ce pour des raisons philosophiques, la vérité n'existant pas en soi, d'une façon immuable, ni pour moi ni pour les autres; et pour des raisons humaines : les savants manquent de contradictions, manquent d'erreurs, manquent de vie. Ça, c'était réglé ! Mais j'étais une petitebourgeoise, fille de bourgeois et, à ce moment, femme d'un intellectuel petit-bourgeois, soucieuse "d'esthétisme" et d'une certaine forme de 
réussite sociale. C'est au sein de cette réalité que j'ai subi le choc de la remise en question qu'entraînent forcément, pour les personnes dans ma condition sociale, les exigences que posent les prémisses de la conscientisation. C'est au cours d'une session de sensibilisation à la conscientisation en $1979^{1}$ que j'ai compris que j'avais le choix entre foncer tête baissée dans le doute, la peur, les contradictions, ou mourir dans la construction acharnée d'un plan de carrière, cachée derrière des conventions, déguisée à même de belles théories choisies à travers une panoplie de tendances, certaines progressistes, toutes plus exotiques et séduisantes les unes que les autres.

Depuis 1976, j'étais membre du Regroupement des organisatrices et organisateurs communautaires du Québec (devenu plus tard le Collectif québécois de conscientisation). J'étais à ce moment-là militante au Centre de santé pour les femmes de Québec et j'avais tenté, lors des deux années précédentes, de conjuguer la conscientisation avec le féminisme, à travers une expérience d'enseignement et de consultation de stage en organisation communautaire à l'Université Laval. C'était clair quand je me suis présentée à la sélection au C.L.S.C. La Source, et c'est sur cette base qu'on m'a engagée.

\section{Un C.L.S.C. en phase d'organisation}

Le C.L.S.C. La Source était à ce moment en pleine phase d'organisation. Il n'avait pas encore les locaux définitifs qu'il a aujourd'hui et n'avait pas encore vécu les transferts de personnel du Département de santé communautaire, encore moins ceux du Centre de services sociaux et de la Commission de la santé et de la sécurité du travail. Nous étions très peu d'intervenantes et intervenants, et la majorité d'entre nous étions des intervenants sociaux dont un organisateur communautaire, une travailleuse communautaire, deux agents de relations humaines et une seule infirmière en santé communautaire. Les choses ont bien changé depuis. Au moment où je rédige cet article, les intervenantes et intervenants sociaux sont minoritaires par rapport aux professionnelles et professionnels de la santé, même si l'équipe s'est adjoint quatre autres techniciennes en assistance sociale, en comptant les contractuelles. II y a trente-deux intervenantes et intervenants en santé. Le C.L.S.C. est divisé en trois modules : administration, social et santé. Son personnel compte en tout quatre-vingt-deux membres, dont soixante-dix-sept syndiquées(és) dans quatre syndicats différents.

À mon arrivée au C.L.S.C., en janvier 1983, il était entendu que je prendrais un moment pour connaître le milieu; suite à quoi nous élaborerions, l'organisateur communautaire, Louis Rouillier, et moi, un 
plan d'action qui tiendrait compte des trois mandats qui nous étaient confiés, soit :

- développer de nouvelles actions prioritaires;

- assurer les services courants aux groupes du milieu ;

- assurer les services courants au C.L.S.C.

\section{Un milieu et son vécu : priorité aux sans-emploi}

Les groupes du milieu se sont révélés être en majorité des groupes de loisirs et de services. Certains, sans que cela se traduise dans l'action, se définissaient comme des groupes de pression : un comptoir alimentaire, une maison de jeunes, un groupe écologique, quelques associations de locataires, un groupe de personnes âgées différent de ceux de l'"Âge d'or", quelques groupes de femmes, dont une AFÉAS, et un groupe de familles monoparentales.

Par ailleurs, le problème du chômage s'amplifiait sur notre territoire comme ailleurs : au bureau du Ministère de la main-d'oeuvre et de la sécurité du revenu à Charlesbourg, qui dessert le Grand Charlesbourg, une partie de Limoilou, Saint-Émile et Sainte-Brigitte-de-Laval, de 3600 chèques émis en 1982, on était passé à 4600 en 1983 et à plus de 5200 à l'été 1984. On pouvait aussi se rendre compte que le Nord du territoire desservi par le C.L.S.C. La Source (Lac-Saint-Charles, les Cantons-Unis de Stoneham et Tewkesbury, Saint-Adolphe et NotreDame-des-Laurentides) avait un plus fort taux de chômage et était plus pauvre en services et développement que le Sud (Charlesbourg et Orsainville); quoiqu'au Sud on pouvait observer des concentrations de logements à prix modiques, souvent en retrait des centres d'activité urbaine. On pouvait supposer qu'il y avait là plusieurs femmes cheffes de famille, assistées sociales, probablement plus jeunes que celles du Nord. Ces arguments nous permirent de justifier une action prioritaire avec les personnes sans emploi. Outre le groupe Entraide qui rassemblait des jeunes se faisant des emplois temporaires à même les programmes gouvernementaux, il n'existait aucune ressource pour les sans-emploi et la défense de leurs droits sociaux. Nous prévoyions travailler séparément avec les femmes et les hommes. Nous étions convaincus qu'être sans emploi ne signifiait pas la même chose pour un homme et pour une femme et que ça ne se vivait pas de la même façon. Nous faisions l'hypothèse que les femmes étaient plus souvent assistées sociales que chômeuses, qu'elles l'étaient souvent devenues suite à un divorce et qu'elles se retrouvaient, par conséquent, seules avec de lourdes charges familiales sans être nécessairement ni disponibles, ni préparées à l'emploi. 
Pour leur part, les hommes devaient être plus démolis dans leur image sociale par le statut de chômeur ou d'assisté social, à cause du rôle de pourvoyeur que leur attribue culturellement notre organisation sociale. Tous deux devaient cependant connaître l'isolement, la honte et la peur devant les préjugés sociaux et le harcèlement légal des différents agents d'aide sociale et de chômage. Tous deux devaient vivre une certaine forme d'aliénation dans leurs différences culturelles de sexe et dans leurs ressemblances culturelles de classe.

C'est donc la moitié de notre temps/travail que chacun et chacune accorderions cette année-là à ce dossier prioritaire, tout en continuant à assurer, pour l'autre moitié, les services courants aux groupes du milieu et au C.L.S.C. Pour ces autres mandats, nous prévoyions répondre à toutes les demandes par différentes interventions, allant de la référence à la consultation ou à l'engagement ponctuel selon certains critères que nous continuons à ajuster. Nous nous sommes partagé les représentations sur les comités susceptibles de réunir différents groupes du milieu.

C'est encore de cette façon que nous travaillons, en réajustant constamment nos critères et nos outils de travail à la lumière des évaluations annuelles que nous faisons avec la coordonnatrice de notre module.

\section{Prête, pas prête, j’y vas : les débuts de l'action avec les femmes}

Le 26 avril 1983, à peine quatre mois après mon engagement comme travailleuse communautaire au C.L.S.C. La Source, se tenait à Notre-Dame-des-Laurentides, dans une des classes de la vieille école Assomption, la première réunion des femmes sans emploi. Une semaine plus tôt, Jacqueline et Marguerite, deux assistées sociales et moi, étions allées suivre la session sur la Loi d'aide sociale ${ }^{2}$ à l'Association pour la défense des droits sociaux du Québec métropolitain, l'A.D.D.S.Q.M.

Ce n'était pas le moment idéal pour commencer. Je n'étais pas vraiment prête. J'aurais voulu réunir plus de femmes, une de chaque localité. J'aurais aimé m'outiller davantage au niveau de la planification. Mais l'occasion de suivre le cours à l'A.D.D.S.Q.M. se présentait à ce moment précis et elle était trop belle : il fallait la prendre, dépasser les limites et les peurs liées à cette question de timing et plonger... prête, pas prête! Cette expérience, très importante pour nous trois, fut certainement déterminante dans l'orientation de R.Q.S.E. du Nord. 
Marguerite et Jacqueline, que j'étais allée chercher dans leur cuisine, comme elles le disent elles-mêmes, ainsi que Louise, ex-assistée sociale, travailleuse sur un programme d'emploi temporaire, avaient accepté de s'engager dans une démarche de six semaines (qui en a duré huit en réalité et s'est terminée à six femmes) pour jeter les bases d'un regroupement qui leur ressemble.

\section{Jeter les bases avec les femmes}

J'avais prévu, pour cette phase que je voulais "planificatrice", un outil global. Il s'agissait d'un immense tableau en blanc qui recouvrait presque tout un mur.

Quatre grands titres en tête d'affiche:

- Pourquoi on veut se regrouper? (objectifs)

- Avec qui on veut se regrouper? (mobilisation)

- Qu'est-ce qu'on va faire? (activités)

- Comment on va s'y prendre? (moyens)

Cet outil, je l'avais fabriqué à partir de la grille de préparation d'une activité de l'Organisation populaire des droits sociaux de Mercier. ${ }^{3}$ D'autres outils ont été introduits, au besoin, pour faciliter la prise de parole, alimenter un nouveau thème, permettre des synthèses. Au cours de cette étape, les femmes ont surtout pris la parole sur ce qu'elles vivaient, comment elles voyaient la vie, leur vie, la société, etc. On se réservait un moment pour essayer de trouver des réponses aux questions qu'elles apportaient concernant l'aide sociale, le divorce, les rentes et le testament. Je tenais un journal de bord très détaillé auquel les femmes ont toujours eu accès. Il était plein de photos, de tableaux réduits, de cartes de souhaits et de lettres adressées au Regroupement. Aujourd'hui, il a cédé la place au cahier des procès-verbaux dont la rédaction est partagée, sur une base volontaire, par les femmes du comité de coordination. Pendant ces huit semaines de planification, les femmes ont vraiment décidé des objectifs du Regroupement et de sa base sociale. "Ce qu'on ferait " et "comment on le ferait" restaient à construire ensemble dans la pratique et la réflexion.

Les objectifs de R.Q.S.E. du Nord sont, depuis, demeurés les suivants : briser notre isolement ; connaître et défendre nos droits dans l'aide sociale et le divorce, individuellement et collectivement; nous entraider ; faire un pas ensemble vers notre autonomie. 


\section{Se regrouper avec des femmes "comme nous": une option de femmes et de classe}

À la question "qui on veut regrouper ?", on avait donné la réponse suivante : des femmes du Grand Charlesbourg, de tous âges, qui vivent de prestations de l'État (assistées sociales, chômeuses, retraitées) ou de petites pensions alimentaires comblées par l'aide sociale, quel que soit leur statut civil (divorcées, mariées, vivant en union libre, célibataires, séparées ou veuves). Pourtant, ce choix de se regrouper avec des "femmes comme nous" n'avait pas été facile à faire. Quand elles ont fait leur portrait collectif, ${ }^{4}$ elles se sont rendu compte qu'elles avaient toutes des problèmes de pauvreté et des problèmes "spéciaux" de femmes. À travers les discussions, elles ont vite compris que, pour remonter aux causes de ces problèmes et y apporter des solutions (pas juste pour une - ça ne règle rien au fond - mais pour toutes), il fallait connaitre plein de choses sur les lois, le fonctionnement des organisations, les réseaux des services publics, la "politique», etc. Les grands mots étaient lancés. Réaction spontanée : il nous faut, dans notre Regroupement, des personnes qui "savent " : des avocates, des femmes instruites ou, tout au moins, des conférencières qui viendraient périodiquement nous dire, nous enseigner. Les femmes se référaient spontanément au seul modèle d'éducation qu'elles connaissent, celui que Freire appelle l'éducation bancaire. ${ }^{5}$

"Décide donc toi Jocelyne - nous autres on n'est pas habituées dans ces affaires-là - Pis, ça nous fatigue - continue, on va te suivre - on sait que t'as étudié longtemps là-dedans pis, qu'on peut te faire confiance : $t$ ' es une femme, $t$ ' as vécu, pis t' es de notre bord...".

J'avais aussi fait mon portrait ${ }^{4}$ au cours de la démarche et ça nous avait permis de cerner nos différences culturelles, économiques et autres, et aussi nos ressemblances, qui se situaient surtout aux niveaux des responsabilités et de certains traits culturels dits "féminins". J'aìeu. à me situer par rapport à ce type de réactions. Ce fut l'occasion d'une amorce d'analyse sociale que nous avons poursuivie quand nous avons préparé la session sur la Loi d'aide sociale "pour des femmes".

Nous avons fabriqué une pyramide sexuée des classes sociales ${ }^{6}$ dans laquelle nous nous sommes situées à partir de nos photos et de notre fonction sociale. Nous y avons également placé plein de photos de personnes qu'on connaissait. La pyramide des classes sociales est un outil visuel et souvent "tactile" auquel nous avons ajouté un élément nouveau. Une ligne plus oblique que verticale la divise inégalement entre hommes et femmes, le bas de la pyramide étant forcément plus large, plus rempli de femmes tandis que le haut, comme nous le savons 
toutes, est plus réduit, étant presque vide de femmes. Cet outil constitue, en quelque sorte, notre cadre d'analyse, et on s'en sert périodiquement pour comprendre, pour décider, pour se faire une opinion. Au Regroupement des femmes sans emploi, on continue, jour après jour, à essayer de comprendre les intérêts qu'on a en tant que femmes de la classe populaire. Et les deux éléments, "femmes" et "classe populaire", sont aussi importants l'un que l'autre. Ce qui est clair, pour les femmes du Nord, c'est que, pour améliorer nos conditions de vie, on a intérêt à lutter autant dans nos vies privées qu'à l'extérieur pour amener des changements dans les rapports de classe et de sexe également. Par exemple, on sait que si demain matin tous les ministres étaient des femmes, ça ne changerait pas nécessairement nos conditions d'assistées sociales. Il faut s'en occuper nous-mêmes avec d'autres groupes d'assistées sociales et sociaux, de chômeuses et chômeurs et de syndiquées(és). On sait aussi que si la classe populaire avait plus de pouvoir, si on vivait dans une organisation socialiste, ça n'égaliserait ni n'améliorerait nécessairement les rapports hommesfemmes. Il faut s'en occuper nous-mêmes avec les autres groupes de femmes qui nous ressemblent. Sans que ce soit dit ainsi, on reconnaissait et abordait à la fois l'oppression de classe et l'oppression de sexe.

\section{R.Q.S.E. du Nord : des activités, une organisation}

Depuis l'été 1983, bien des activités ont été entreprises; elles ont été préparées, exécutées et évaluées avec les femmes: trois sessions sur la Loi d'aide sociale adaptées à la réalité des femmes assistées sociales, des visites à des groupes de différentes régions, des cafésrencontres sur des thèmes choisis par les femmes (sexualité, religion, peur, relations avec les hommes, éducation des enfants, la santé, le Livre blanc, etc.), des fêtes, deux 8 mars chez nous et en région, une participation active à la semaine des assistées sociales et sociaux de 1985 et, depuis septembre 1984, des services d'aide aux femmes en difficulté en rapport avec l'aide sociale et le divorce (rencontres collectives et téléphone).

Le groupe s'est incorporé dès l'automne 1983 ; il a son propre local depuis l'hiver 1984 et s'est donné des structures qui se modifient au besoin, de trimestre en trimestre. Une seule constante, c'est le comité de coordination qui est formé à l'heure actuelle de neuf femmes sans emploi et de la personne-ressource. Ce comité a tous les pouvoirs et... toutes les responsabilités. Ces femmes partagent la responsabilité des trois jours de permanence au local, du courrier et de l'information, de 
l'accueil des nouvelles, des rencontres collectives, de la rédaction du journal, de l'organisation des activités spéciales et des finances.

\section{Un premier bilan : changement personnel et action collective}

\section{Qui change qui ? et quoi?}

À l'occasion d'une session de formation du Collectif québécois de conscientisation (C.Q.C.) à laquelle je participais en avril 1984 avec Marlène, une militante de R.Q.S.E. du Nord, nous avons eu l'occasion de faire un exercice qui nous a permis de vérifier les changements chez les personnes. Nous avons tenté de situer les écarts entre "les portraits" de la femme de la classe ouvrière et de la militante petite-bourgeoise, au début du groupe et après un an de fonctionnement (Tableau 1). Ces portraits ne sont ni complets, ni définitifs; nous avons même l'intention de les refaire toutes ensemble lors de la prochaine journée d'évaluation : ils révéleront certainement d'autres changements. D'autant plus que d'autres femmes, plus jeunes et avec une culture différente, se sont jointes à R.Q.S.E. Cependant, ces portraits constituent, croyons-nous, un indice de la grande capacité de changement des femmes engagées dans un processus de conscientisation. Ils révèlent aussi la disponibilité à l'interpellation et au changement personnel de la personne-ressource, afin que se dégagent enfin "des ponts" entre militantes de la classe populaire et militantes petites-bourgeoises. Dans sa vie d'éducatriceéduquée, l'intervenante qui a pris la position d'alliée des femmes de la classe populaire, si elle est conséquente avec cette approche dialectique qu'est la conscientisation, doit elle aussi se laisser interpeller par ces femmes de la classe populaire, tant dans sa culture de femme petitebourgeoise que dans sa situation de classe. ${ }^{7}$ 
TABLEAU 1

\begin{tabular}{|c|c|}
\hline \multicolumn{2}{|c|}{ Femme de la classe populaire } \\
\hline Au début & $\begin{array}{l}\text { Maintenant } \\
\text { (après un an) }\end{array}$ \\
\hline Manque de confiance en soi & $\begin{array}{l}\text { - Confiance en soi dans le groupe } \\
\text { (prise de parole) } \\
\text { - On peut tout faire (ne voit pas } \\
\text { toujours les limites) }\end{array}$ \\
\hline $\begin{array}{l}\text { On préfère plutôt parler que lire et } \\
\text { écrire }\end{array}$ & $\begin{array}{l}\text { - On lit plus (aide sociale, divorce, } \\
\text { comité de lecture) } \\
\text { - On écrit plus (rapports, corres- } \\
\text { pondance) } \\
\text { - On parle plus à la lumière d'infor- } \\
\text { mations }\end{array}$ \\
\hline $\begin{array}{l}\text { Culpabilisation au niveau des res- } \\
\text { ponsabilités familiales, situation d'as- } \\
\text { sistée sociale }\end{array}$ & $\begin{array}{l}\text { - Léger dégagement de cette cul- } \\
\text { pabilisation (vie quotidienne et } \\
\text { cafés-rencontres) }\end{array}$ \\
\hline $\begin{array}{l}\text { On a trop de principes (moraux et } \\
\text { religieux) }\end{array}$ & $\begin{array}{l}\text { - Clarification et plus de respect } \\
\text { pour les autres et leurs différences }\end{array}$ \\
\hline $\begin{array}{l}\text { Importance de se retrouver dans } \\
\text { des activités d'entraide (accompa- } \\
\text { gnement, soutien) }\end{array}$ & $\begin{array}{l}\text { - Même importance, doublée d'un } \\
\text { désir d'aborder concrètement la } \\
\text { défense des droits }\end{array}$ \\
\hline $\begin{array}{l}\text { Recours à des groupes traditionnels } \\
\text { ou de relations humaines }\end{array}$ & $\begin{array}{l}\text { - Engagement dans un regroupe- } \\
\text { ment plus militant (rupture avec } \\
\text { certains réseaux anciens) }\end{array}$ \\
\hline Pas concernées - fatalité & $\begin{array}{l}\text { - Peur de trop comprendre, de } \\
\text { devoir aller plus loin, }{ }^{8} \text { de se cou- } \\
\text { per de sa famille, de ses amis }\end{array}$ \\
\hline $\begin{array}{l}\text { Quilles, tricot, tissage, très impor- } \\
\text { tants }\end{array}$ & $\begin{array}{l}\text { - Ne prennent plus autant de place; } \\
\text { souci d'apprendre à travers les } \\
\text { "loisirs" }\end{array}$ \\
\hline Maladies physiques, psychologiques & $\begin{array}{l}\text { - Maladies mieux assumées; on } \\
\text { veut se donner du bon temps; } \\
\text { meilleure santé mentale }\end{array}$ \\
\hline $\begin{array}{l}\text { Importance de l'habillement adapté } \\
\text { aux situations }\end{array}$ & $\begin{array}{l}\text { - Plus de liberté, moins de conven- } \\
\text { tion par rapport à l'habillement }\end{array}$ \\
\hline $\begin{array}{l}\text { Démarche volontariste (le but est } \\
\text { absolutisé) }\end{array}$ & $\begin{array}{l}\text { - Le but est relativisé (l'important } \\
\text { c'est d'être en route; plus d'atten- } \\
\text { tion à ce qui se passe, au rythme } \\
\text { des autres, au nôtre, etc.) }\end{array}$ \\
\hline
\end{tabular}


TABLEAU 1 (suite)

\begin{tabular}{|c|c|}
\hline \multicolumn{2}{|c|}{ Militante petite-bourgeoise } \\
\hline Au début & $\begin{array}{l}\text { Maintenant } \\
\text { (après un an) }\end{array}$ \\
\hline Approche plus théorique & $\begin{array}{l}\text { - Approche plus à partir de la réalité, } \\
\text { de la pratique }\end{array}$ \\
\hline Militante féministe petite-bourgeoise & $\begin{array}{l}\text { - Meilleure intégration dans une } \\
\text { militance plus large et reconnais- } \\
\text { sance de mêmes éléments de cul- } \\
\text { ture de femme dans les milieux } \\
\text { populaires } \\
\text { - Reconnaissance d'autres éléments } \\
\text { de culture de femme dans les } \\
\text { milieux populaires et qui font } \\
\text { qu'il y a des tabous (avortement, } \\
\text { religion) ou des incompréhensions } \\
\text { de fond (pornographie, violence) }\end{array}$ \\
\hline $\begin{array}{l}\text { Fort sentiment de responsabilité, de } \\
\text { maternage }\end{array}$ & $\begin{array}{l}\text { - Un peu de distanciation (plus de } \\
\text { confiance au groupe) }\end{array}$ \\
\hline $\begin{array}{l}\text { Peu d'importance accordée aux } \\
\text { conventions dans l'habillement }\end{array}$ & $\begin{array}{l}\text { - Attention pour "honorer", par } \\
\text { exemple par l'habillement, les } \\
\text { femmes des milieux populaires } \\
\text { (fêtes, visites) }\end{array}$ \\
\hline En dormait mal la nuit & $\begin{array}{l}\text { - Se donne un peu plus le droit et } \\
\text { les moyens de vivre pour elle }\end{array}$ \\
\hline
\end{tabular}

\section{"On est tannées de placoter"}

En mai 1984, nous avons évalué notre démarche et établi ensemble une planification qui se situait dans un objectif général de mobilisation interne et externe, dans la perspective d'ouvrir R.Q.S.E. du Nord à d'autres femmes et de renforcer notre noyau dur. "On est tannées de placoter. On dit qu'on veut accueillir des femmes en difficulté sans encore le faire. Il faut se former, ça nous prend d'autres femmes avec nous, mais il faut foncer. Ouvrons! „Pour se préparer et y arriver, nous nous étions donné cinq objectifs vérifiables en décembre 1984 :

- avoir atteint vingt-cinq femmes, par nos services, en décembre ;

- avoir grossi le noyau dur de deux femmes ;

- apprendre à réagir à ce qui nous touche, sur le territoire et comme femmes sans emploi ; 
- développer des alliances avec les groupes qui nous ressemblent ;

- assurer nous-mêmes notre organisation.

Les moyens qu'on comptait prendre passaient par les activités suivantes :

- campagne de publicité dans les paroisses;

- contacts personnels et chaleureux avec des femmes en difficulté ;

- cours sur la Loi d'aide sociale, en novembre ;

- comité de lecture de l'actualité avec Noëlla, notre stagiaire ;

- préparation de visites à la Maison des femmes de Québec, au Centre des femmes de la Basse-Ville et à l'A.D.D.S.Q.M. ;

- partage des trois jours de permanence et des responsabilités entre les femmes du noyau dur;

- journée de formation sur la permanence (activité de fin de stage de Noëlla).

Nous avons pu constater, en décembre, que les femmes en difficulté qui nous avaient téléphoné (vingt-huit) demandaient de l'aide surtout par rapport au divorce et qu'elles étaient presque toutes assistées sociales ou en voie de l'être. Ce ne sont cependant pas ces femmes, même si elles ont participé à une rencontre collective, qui viennent se joindre au noyau dur, à moyen terme en tout cas. En effet, celles qui reviennent au Regroupement, ce sont les femmes qui suivent le cours sur la loi 26 et qui étaient déjà en contact avec l'une ou l'autre femme du comité de coordination. En effet, sur les quinze femmes inscrites au cours, huit se sont présentées, six l'ont terminé et quatre se sont jointes au noyau dur, partageant les responsabilités de la permanence. Ces quatre femmes avaient été amenées par l'une ou l'autre membre du comité de coordination. Les femmes qui viennent militer au Regroupement, ce sont celles qui ont gagné une lutte avec R.Q.S.E. du Nord ou qui sont en attente d'une décision de la Commission des affaires sociales parce qu'elles y sont allées en appel. Jusqu'à maintenant, l'aide sociale parait plus mobilisante, quoiqu'on reçoive plus de demandes d'aide par rapport au divorce. Plusieurs facteurs peuvent expliquer cette observation. Les plus évidents sont que le divorce est plus complexe sur le plan légal et qu'il est tellement perturbant émotivement qu'il ne correspond que très rarement aux conditions d'un engagement social, tout ceci restant à vérifier à long terme.

Au cours de l'année, nous avons constaté davantage l'écart qui séparait déjà les femmes formées pour donner le cours de celles qui le reçoivent, celles formées pour le comité de coordination et toute autre responsabilité et les nouvelles qui ne sont pas prêtes aux discussions de 
fond, à la libre expression, à la confrontation des idées, aux informations de base, etc. C'est là tout le problème de la relève dans ce type de groupe d'action. Les nouvelles n'ont pas eu la chance de cheminer dans le programme initial dont le rythme était plus lent. Nous avons donc réajusté notre programme et introduit un après-midi par semaine pour accueillir les nouvelles. Au début, ces après-midi ressemblaient un peu à nos rencontres collectives. Depuis le 8 mars, influencées par les femmes du Centre des femmes de la Basse-Ville, on utilise le théâtre pour s'exprimer et discuter sur des thèmes choisis par chacune des participantes.

Le comité de lecture, dont s'occupe Noëlla, nous a permis de reconnaître l'importance de choisir ce qu'on lit, de choisir la source de notre information, de lire en profondeur, de savoir faire les liens et de lire entre les lignes. Depuis, les femmes ont choisi de se donner des cafés-rencontres sur l'actualité comme, par exemple: "Ça veut dire quoi le Livre blanc sur la fiscalité pour nous les femmes assistées sociales?".

Indépendamment de ce qu'on avait prévu, nos alliances et, par conséquent notre esprit de lutte, se développent à travers certains événements tels que: consultation sur les problèmes que vivent les femmes, le 8 mars, la visite de femmes de pays en voie de développement, le dépôt du Livre blanc sur la fiscalité, la semaine des assistées sociales et assistés sociaux, etc. De plus en plus, on nous invite dans les groupes populaires, on nous demande des appuis. Au Regroupement, on a souvent à consulter l'A.D.D.S. pour des questions d'aide sociale. On suit leur lutte sur le montant attribué pour la rentrée scolaire. On a participé à la lutte lancée par l'O.P.D.S. de la région de Montréal sur la discrimination faite aux assistées sociales et aux assistés sociaux dans certaines caisses populaires. On a attendu la décision de la Commission des affaires sociales sur l'appel qu'a placé Jeannette, une femme de notre Regroupement, concernant un montant global obtenu lors d'un règlement de divorce qui, parce qu'il lui est versé périodiquement, lui est coupé de l'aide sociale. ${ }^{10}$ On a participé à certaines activités de solidarité internationale au Centre des femmes de la Basse-Ville. C'est beaucoup à travers les alliances et les services qu'on aborde la lutte.

Les défis qu'on a à relever dans cette pratique de conscientisation sont nombreux. Celui de la permanence quotidienne partagée par les femmes, pour que l'organisation leur appartienne, sans que, pour autant, elles se responsabilisent trop; celui de l'alliance entre petitebourgeoise et femmes de classe populaire, sans pour autant exercer de pouvoir idéologique ; celui de considérer également, dans l'action, les 
deux fronts de lutte, dans le flot des "événements importants" concernant les femmes pauvres (politique familiale, fiscalité, décisions 85 , etc.) ; celui de la continuité, de la constance quand la flamme du début ne vous nourrit plus; celui de croire encore à de nouveaux rapports non hiérarchiques à construire quand, une fois formées dans le groupe, une puis deux femmes vont prendre la présidence d'un organisme; celui d'être patientes, acharnées et solidaires quand il vous semble que rien ne change. Le changement individuel profond (et, d'autant plus, le changement collectif profond) prend beaucoup de temps. Des femmes qui ont souvent investi plus de la moitié de leur vie dans certaines valeurs qu'on sait aliénantes peuvent-elles facilement s'en libérer quand elles en deviennent conscientes? Les solutions de rechange, les alternatives sont souvent bien rares et les espoirs bien minces, ou "spirituels", pour certaines femmes de la classe populaire. À ce propos, il faut réfléchir sur notre pratique à la lumière d'une grille d'analyse telle que le propose les auteures de : Nous, notre santé, nos pouvoirs dans leur tableau: "Analyse comparative des coûts et bénéfices des valeurs traditionnelles et féministes". ${ }^{11}$

Restent des éléments concrets qui freinent la mobilisation et avec lesquels il faut composer quotidiennement : les problèmes de transport (on n'est pas en ville!), de garderie, la fournaise qui saute en plein hiver (déménagerons-nous?), la permanence qui devient plus difficile l'hiver. Et les problèmes encore plus profonds qui touchent de près la mobilisation; les femmes, les plus vieilles surtout, qui s'appauvrissent de plus en plus dans ce contexte de crise économique qui les touche particulièrement. Le travail au noir, les " jobbines" d'entretien ménager, d'artisanat, de garde d'enfants qui se développent alors que la santé se détériore et que la lutte deviendrait d'autant plus importante : paradoxe de la lutte. La mobilisation en souffre... elle en souffre partout.

\section{Conclusion R.Q.S.E. du Nord et le mouvement féministe}

L'heure ne semble pas être à la mobilisation au Québec; on a plutôt l'impression qu'elle est à la conciliation ou à la défaite. Les vieux partis politiques prennent le dessus, les médias sont de plus en plus faussement neutres, les éléments de gauche sont plus facilement cassables dans la division que créent, de connivence, les grands patrons et l'État. Des événements se produisent, qui n'auraient même pas été pensables dans les années 1970 - - la visite de Reagan à Québec, 
par exemple. Dans le milieu artistique, rarissimes sont celles et ceux qui résistent. La mobilisation est difficile. On pourrait s'en tenir à cette constatation... Pourtant, à y regarder de plus près, il se passe des choses importantes au Québec actuellement. De plus en plus de groupes de femmes de la classe populaire se forment, brisant leur isolement et leur silence. La "rage" des jeunes gronde; ils manifestent leur colère, leur haine et leur désespoir. Les sans-emploi s'organisent ; les syndicats s'en préoccupent, les groupes qui font de la formation populaire aussi. Certains syndicats locaux mènent des luttes originales sur des enjeux qui dépassent l'intérêt immédiat des travailleuses et travailleurs concernés: "Pour créer de nouveaux emplois décents!". Des intellectuelles(Is) de toutes disciplines, plus seulement des sociologues, travailleurs sociaux et psychologues mais aussi des économistes, écologistes et professionnelles (Is) de la santé se joignent à des actions populaires sur des terrains différents ou poursuivent des recherches dans lesquelles des prises de position en faveur des opprimés sont évidentes. Pensons aux économistes Lise Poulin-Simon et Diane Bellemare, ${ }^{12}$ qui poursuivent une recherche très engageante sur la politique du plein emploi. Pensons aussi à la Faculté d'éducation permanente de l'Université de Montréal qui a affecté une chercheuse à la recherche de I'AFÉAS sur la situation des femmes au foyer. ${ }^{13}$

Les fronts de lutte se reconnaissent enfin et s'appuient mutuellement. Ils tentent de dégager des pistes communes. Cette année, le comité organisateur du 8 mars, à Québec, était composé de représentantes de groupes autonomes de femmes, de groupes syndicaux et de groupes de solidarité internationale des femmes. Elles ont organisé, entre autres, une caravane pour aller chercher les femmes des groupes, en région. On en parle encore à R.Q.S.E. du Nord. La semaine des assistées sociales et sociaux a intégré cette année la fête du $1^{\text {er }}$ mai. Une belle occasion d'élargir nos revendications jusqu'au plein emploi et d'aller chercher l'appui des travailleuses et travailleurs.

On se respecte aussi davantage. On comprendra facilement que certains groupes ne soient actuellement pas prêts à appuyer concrètement une telle lutte. On se menace moins. On sent la nécessité de se serrer les coudes, de développer une stratégie concertée pour faire face à la tendance généralisée adoptée d'emblée par la partie patronale de "diviser pour mieux régner".

\section{"Tant qu'il y aura une femme qui crie..."}

Le mouvement féministe est sûrement un des mouvements sociaux les plus importants que l'humanité ait connu dans son histoire. Le 
simple fait qu'il concerne la moitié du monde opprimée par l'autre moitié est d'une signification évidente et irréfutable. Être féministe signifie, sur le plan universel, que tant et aussi longtemps qu'il y aura une injustice faite à un groupe de femmes à travers le monde, il faudra la dénoncer et s'allier à la lutte contre cette injustice. Le mouvement de libération des femmes est, bien sûr, porteur de libération pour l'humanité entière, de la même façon que l'est le mouvement de libération des masses populaires. Et les injustices faites aux femmes le sont doublement dans la classe populaire.

Le mouvement féministe, jusqu'à maintenant porté par la petitebourgeoisie, perce actuellement dans les classes populaires. De plus en plus de groupes de femmes de classe populaire s'organisent, souvent amenés par des intervenantes féministes socialistes. De plus en plus de groupes populaires composés en majorité de femmes analysent leur pratique à la lumière de l'analyse féministe. Je pense particulièrement aux groupes d'assistées sociales qui font actuellement une analyse féministe du Livre blanc sur la fiscalité, à travers le Québec. Cette percée que fait actuellement le mouvement féministe dans les classes populaires est d'une très grande importance dans la perspective d'un projet de société porté par des femmes et des hommes de la classe populaire.

\section{L'approche conscientisante féministe pour la transformation des structures et des mentalités}

Dans un document de travail que je présente avec son accord, Gisèle Ampleman, femme de longue expérience de pratiques de conscientisation et co-auteure de Pratiques de conscientisation, définit ce qu'est l'approche conscientisante féministe pour la transformation des structures et des mentalités. La visée qu'elle présente est d'envergure politique, à la fois souple et clairement orientée. Une visée qui porte en elle-même la nécessité de couvrir les deux fronts de lutte dans le dialogue. On y retrouve, clairement énoncés, les éléments qui permettent de comprendre à quel niveau de conscience se situent une femme et un groupe de femmes de classe populaire (aux plans historique, économique, psychologique, culturel, religieux et politique). Mis à part la réserve que j'ai, en tant que non-croyante, sur le thème de la foi, mais tout en composant avec cette dimension présente dans la culture des femmes de classe populaire, cette grille m'apparaît être un outil précieux. Non seulement elle nous permet de penser avec clarté la libération de la double oppression concrète de la majorité des 
femmes, mais elle nous permettra de nous en servir pour clarifier, évaluer, aller plus loin dans nos groupes et avec d'autres groupes et intervenantes.

\section{L'approche conscientisante féministe de la classe populaire pour la transformation des structures et des mentalités}

Visée : Devenir des femmes libres et autonomes, porteuses et créatrices de l'Histoire, pour la construction d'une société égalitaire, démocratique, socialiste, autogestionnaire.

Au plan de l'histoire : se réapproprier son histoire personnelle et collective (histoire située et datée).

Passer d'une conscience a-historique à une conscience historique personnelle et collective

- comme femme dans son histoire personnelle,

- comme femme appartenant à la classe populaire,

- comme femme appartenant au mouvement populaire, au plan local, régional, national et international,

- comme femme appartenant au mouvement féministe, au plan local, régional, national et international.

Au plan économique : Se libérer des exploitations économiques capitalistes et patriarcales.

Passer d'une conscience dépendante à une conscience autonome

- éliminer la division sexiste du travail,

- avoir un travail rémunérateur : travail extérieur et/ou travail domestique,

- arriver à vivre une autonomie financière,

- travail égal = salaire égal,

- revendiquer ses droits comme travailleuse.

Au plan psychologique : devenir une femme libre et autonome.

Passer d'une conscience coupable à une conscience libérée féministe

- développer l'estime de soi,

- développer sa capacité de s'affirmer,

- prendre la parole, dire son vécu comme femme et comme femme appartenant à la classe populaire,

- savoir exprimer ses besoins, ses désirs, ses sentiments et ses émotions, reconnaître son droit au plaisir,

- se réapproprier son corps,

- se réapproprier son identité individuelle et collective, comme femme appartenant à la classe populaire féministe. 
Au plan culturel: se libérer des aliénations culturelles et religieuses, capitalistes et patriarcales.

Passer d'une conscience soumise à une conscience critique libératrice (tenir compte de l'univers mythique et symbolique ainsi que du langage et de la culture populaires)

- se libérer des préjugés, de la honte et des peurs,

- se libérer des stéréotypes sexistes,

- se libérer des rôles traditionnels,

- se libérer des modèles dominants (masculins et religieux),

- se libérer d'une morale et d'une religion culpabilisantes et individualistes,

- se libérer d'une religion aliénante, dominante et sexiste,

- inventer de nouveaux modèles et de nouveaux rôles propres à sa culture populaire et à sa foi,

- se réapproprier les réalités d'une foi libératrice,

- se réapproprier son univers culturel et religieux en fonction de ses intérêts de femme appartenant à la classe populaire féministe.

Au plan politique: se libérer des dominations politiques, capitalistes et patriarcales.

Passer d'une conscience fataliste à une conscience politique

- prendre des décisions en fonction de son "JE", comme femme de la classe populaire féministe,

- prendre des décisions en fonction d'un "NOUS" populaire féministe,

- établir des rapports égalitaires dans le quotidien et dans le collectif,

- développer des solidarités entre femmes des classes populaires,

- mener des luttes selon ses propres intérêts de classe populaire féministe,

- développer des alliances avec les "alliées femmes" et avec les "alliés hommes" ayant une même visée politique,

- développer des alliances avec le mouvement des femmes au plan local, régional, national et international.

Créer et vivre des alternatives aux exploitations économiques, aux dominations politiques, aux aliénations culturelles et religieuses.

DEVENIR UNE FORCE DE CHANGEMENT ORGANISÉ PAR LA PRISE DU POUVOIR

SOURCE : G. AMPLEMAN, document de travail non publié, Montréal, avril 1985. 
Souvent suscitée par des intervenantes féministes socialistes, la pratique des groupes de femmes de la classe populaire doit se faire dans le dialogue si on ne veut pas "injecter», comme ont tenté de le faire dans les masses populaires, et avec l'échec qu'on connaît, les marxistes-léninistes, avec leur doctrine de libération tout à fait déconnectée de la culture populaire. La classe populaire est là, majoritaire, comportant deux sexes. Les femmes doublement opprimées sont là, isolées, avec leur vécu, leurs peines, leurs joies, leurs réussites et leurs échecs; comme nous petites-bourgeoises, et autrement. On ne doit surtout pas, comme féministes socialistes, les amener à nous ressembler. Les femmes de la classe populaire ont des éléments très importants à apporter dans la définition d'un projet de société à bâtir ensemble. II faut ouvrir le dialogue puisque nous en avons le pouvoir et qu'elles ne l'ont pas. Utilisons les outils qui permettront de prendre la parole, d'analyser, de collectiviser, d'agir. Passons le savoir que nous avons, mais ouvrons-nous aussi au leur. Apprenons le savoir populaire. Acceptons d'être interpellées, d'être confrontées aussi. Ouvrons-nous aussi au changement... Engageons-nous avec ces femmes dans un processus de conscientisation qui nous mènera là où nous bâtirons ensemble.

\section{Notes et références}

1 G. Ampleman, G. Doré, L. Gaudreau, C. Larose, L. Lebofuf et D. Ventelou, Pratiques de conscientisation, expériences d'éducation populaire au Québec, Montréal, Nouvelle Optique, 1984, pp. 101-131.

2 Il s'agit d'une session conscientisante de vulgarisation de la Loi de l'aide sociale. Elle est offerte par plusieurs groupes d'assistés sociaux au Québec. Elle a été systématisée par Gisèle Ampleman, qui la décrit dans: Pratiques de conscientisation, pp. 41-77.

3 G. Ampleman et al., op. cit., p. 201.

${ }^{4}$ Le portrait est aussi décrit et expliqué dans: Pratiques de conscientisation, pp. 111-112. Pour nous, c'était la "bonne femme". J'y avais ajouté une poche dans le dos pour nos responsabilités, une bulle au-dessus de la tête pour nos rêves et une sacoche pour nos conditions économiques.

5 Paulo Freire, Pédagogie des opprimés, Paris, Maspero, 1980, pp. 50-70.

6 G. Ampleman et al., op. cit., pp. 60-61.

7 Pour bien saisir les définitions de "situation de classe" et de "position de classe", consulter : M. HARNECKER et G. URIBE, Les classes sociales, Montréal, Agence de presse libre du Québec, [1974]. ("Cahiers de formation", 6.3.)

${ }^{8}$ Cette étape fait nettement référence au passage d'un niveau de conscience naïve, presque fataliste, à un niveau de conscience plus critique. Les 
éléments de peur qui sont liés à cette étape réfèrent à la peur de la liberté et aux défis du dépassement de cette peur issue de l'aliénation dont parle Freire.

9 Pour les femmes qui se sont fait dire et qui ont cru toute leur vie qu'elles étaient faites pour enfanter, qui ont eu des grossesses jusqu'à la ménopause ou à l'hystérectomie, le droit à l'avortement est un sujet tabou. Pour des femmes qui ont dû se débarrasser "en vrac" des notions de censure et de péché pour aborder la sexualité, un sujet comme la lutte contre la pornographie est chargé d'incompréhensions fondamentales.

Dans un texte qu'elle a communiqué au Congrès de l'Association internationale des écoles de service social en juillet 1984 et qu'elle a intitulé Une pratique féministe, Pauline Gingras, du C.L.S.C. Basse-Ville, souligne, en utilisant l'exemple de la violence conjugale, la nécessité de bien connaître l'univers thématique des femmes et de se méfier des analyses d'intervenantes sociales féministes petites-bourgeoises (p. 32).

10 Jeannette téléphone au local, le 28 avril 1985. Elle vient de gagner rétroactivement sa cause à la Commission des affaires sociales. Pour elle, ça veut dire la fin des coupures aux trois mois, le droit à la carte pour les médicaments et les besoins spéciaux et un remboursement appréciable pour les trois dernières années. Une belle victoire! À R.O.S.E. du Nord, on fêtera ça et on s'en servira pour d'autres femmes. Au niveau de la lutte, c'est une occasion de passer d'un gain individuel à une lutte collective. Un gain qui nous donne de l'énergie et fait rebondir l'action.

11 C. Corbeil, C. Lazure, G. Legault et A. Paquet, "Féminisme et thérapie vers une nouvelle vision de l'intervention", dans l'ouvrage du GRAFS: Nous, notre santé, nos pouvoirs, Montréal, Albert Saint-Martin/Remue-ménage, $1983: 19-21$.

12 D. Bellemarre et L. Poulin-Simon, Le plein emploi: pourquoi? Montréal, PUQ/IRAT/UQAM (Labrev), 1982.

13 R. Therrien et J.-L. Coulombe, Rapport de l'AFÉAS sur la situation des femmes au foyer, Montréal, Boréal Express, 1984. 